\title{
Generation and Analysis of Genetically Defined Liver Carcinomas Derived from Bipotential Liver Progenitors
}

\author{
L. Zender, ${ }^{*}$ W. Xue, ${ }^{*}$ C. Cordón-Cardo, ${ }^{\dagger}$ G.J. Hannon, ${ }^{*}$ R. Lucito, ${ }^{*}$ S. Powers, ${ }^{*}$ \\ P. Flemming, ${ }^{*}$ M.S. SPECTOR, ${ }^{*}$ AND S.W. LOWE* ${ }^{*} \mathbb{I}$ \\ *Cold Spring Harbor Laboratory, Cold Spring Harbor, New York 11724; \\ ${ }^{\dagger}$ Division of Molecular Pathology, Memorial Sloan-Kettering Cancer Center, New York, New York 10021; \\ ${ }^{\sharp}$ Department of Pathology, Medical School Hannover, Hannover, Germany; \\ "Howard Hughes Medical Institute, Cold Spring Harbor, New York, New York 11724
}

\begin{abstract}
Hepatocellular carcinoma is a chemoresistant cancer and a leading cause of cancer mortality; however, the molecular mechanisms responsible for the aggressive nature of this disease are poorly understood. In this study, we developed a new liver cancer mouse model that is based on the ex vivo genetic manipulation of embryonic liver progenitor cells (hepatoblasts). After retroviral gene transfer of oncogenes or short hairpin RNAs targeting tumor suppressor genes, genetically altered liver progenitor cells are seeded into the liver of otherwise normal recipient mice. We show that histopathology of the engineered liver carcinomas reveals features of the human disease. Furthermore, representational oligonucleotide microarray analysis (ROMA) of murine liver tumors initiated by two defined genetic hits revealed spontaneously acquired genetic alterations that are characteristic for human hepatocellular carcinoma. This model provides a powerful platform for applications like cancer gene discovery or high-throughput preclinical drug testing.
\end{abstract}

Hepatocellular carcinoma (HCC) represents the fifth most frequent neoplasm worldwide. However, due to the lack of treatment options, it constitutes the third leading cause of cancer death ( $>500,000$ deaths per year) (Parkin et al. 2001). The only curative treatment options for HCC are surgical resection or liver transplantation. Unfortunately, at the time of diagnosis the majority of patients present with advanced tumor growth and are therefore not candidates for surgical therapy. Local ablative therapies like transarterial chemoembolization (TACE) or percutaneous ethanol injection (PEI) are being used as treatment options but are only feasible in selected patients and are not curative (Bruix et al. 2004). Systemic chemotherapeutic treatment is ineffective against HCC. For example, whereas doxorubicin or cisplatinum has marginal activity, these agents have no impact on long-term patient survival (Llovet et al. 2003).

More than $80 \%$ of all HCCs in humans are due to infection with hepatitis $\mathrm{B}$ virus (HBV) or hepatitis $\mathrm{C}$ virus (HCV) or aflatoxin B1 (AFB1) intake. The precise mechanisms by which $\mathrm{HBV}$ and $\mathrm{HCV}$ predispose to liver cancer are not fully elucidated. However, it is certain that chronic infection with HBV or HCV leads to a chronic turnover of hepatocytes, and this provides a suitable context for oncogenic genetic alterations. Furthermore, recent studies have shown that the HBx antigen of HBV can bind and functionally inactivate the tumor suppressor p53 (Wang et al. 1994; Ueda et al. 1995; Huo et al. 2001). In fact, mice transgenic for the HBx antigen develop hepatic adenomas and HCC (Ueda et al. 1995). Similar studies have been performed for proteins of $\mathrm{HCV}$. It has been reported that the HCV core protein transcriptionally represses the $\mathrm{p} 53$ promoter in different cell types (Ray et al. 1997). In non-virus-induced liver cancers, p53 mutations may also play a role. For exam- ple, liver cancers arising from aflatoxin exposure frequently have mutations in $\mathrm{p} 53$ at codon 249.

A number of other genetic alterations have been linked to the development of HCC. For example, Rb and/or the insulin growth factor receptor 1 (IGFR1) are frequently mutated or deleted (Murakami et al. 1991; Kim et al. 1996), whereas c-myc and cyclin D1 are often amplified (Peng et al. 1993; Nishida et al. 1994; Kusano et al. 1999; Wong et al. 1999; Niketeghad et al. 2001). Alterations in the Wnt-signaling pathway may also be important during hepatocarcinogenesis. Thus, mutations that activate $\beta$-catenin, or inactivate its negative regulator axin, occur in $30-40 \%$ of human HCCs, and nuclear overexpression of $\beta$-catenin occurs in $10-90 \%$ of these tumors (Nhieu et al. 1999; Buendia 2000; Wong et al. 2001). Deregulation of $\beta$-catenin leads to transcriptional activation of Wnt-pathway target genes like cyclin D1, c-myc, or PPAR $\gamma$, which in turn promote tumor progression by enhancing proliferation.

Despite its impact on human health, liver cancer is understudied compared to other major lethal cancers. In addition, the field has a limited arsenal of model systems in which to study the development of this disease and to test potential intervention strategies. For the most part, the traditional study of human cancers only provides a snapshot of the final result, rather than a detailed roadmap of the route taken by the tumor cell to reach its destination. The ability to model intermediate stages of tumor formation is essential for a complete understanding of the disease - not only in terms of our understanding of disease biology, but also in terms of effectively using biological information for the development of new treatments and therapies.

The use of genetically engineered mouse models to study cancer enables an assessment of how specific ge- 
netic alterations occurring in the human disease affect the tumor phenotype. Currently, mouse models of liver cancer are based mainly on classic transgenic approaches, tetracycline-regulated transgene expression or chemically induced carcinogenesis (Sandgren et al. 1989; Jhappan et al. 1990; Murakami et al. 1993; Verna et al. 1996a,b; Deane et al. 2001; Manickan et al. 2001; Wang et al. 2001; Harada et al. 2004; Shachaf et al. 2004). Although these models have produced important insights into the molecular mechanisms of hepatocarcinogenesis, they have several disadvantages. First, the expression of transgenes by tissue-specific promoters may not target all epithelial cells within the organ (stem-cell and non-stem-cell compartment) and thus may alter the cell of origin from that occurring in the human disease. Furthermore, the expression of the oncogene (or deletion of a tumor suppressor gene) in all cells within a tissue creates a field effect such that all cells have altered gene expression, which does not mimic the situation of spontaneous tumorigenesis and may alter a tumor's evolutionary trajectory. Second, it is very costand time-intensive to generate germ-line transgenic and knockout animals, and production of compound mutant animals can involve complicated intercrossing strategies that are extremely slow. Third, some lesions also produce developmental abnormalities resulting in embryonic lethality or developmental compensation in the resulting tissue, such that the consequences of the mutation may not reflect the acute activation or loss that occurs in tumorigenesis. Obviously, a mouse model that allows bypass of these limitations would be extremely valuable.

Over the last few years, chimeric mouse models based on the genetic manipulation and retransplantation of hematopoietic stem cells have provided new insights into mechanisms of tumor initiation, progression, and treatment response in hematopoietic malignancies (see, e.g., Pear et al. 1998; Schmitt et al. 2002a,b; Hemann et al. 2005). Such models have the advantage of generating genetically defined tumors at a fraction of the time and cost required to produce comparable germ-line models, allowing more genes and gene combinations to be studied. Because the cancers that arise in these animals are derived from targeting stem and/or progenitor cells, they may more accurately reflect the evolution of corresponding human malignancies. Finally, these models are produced by transplantation of genetically altered cells into recipient mice, thereby yielding genetic mosaics where the developing cancer cell is surrounded by otherwise normal counterparts. For example, using such systems, we have shown that mutant Myc alleles obtained from Burkitt's lymphoma are highly oncogenic because they fail to induce the pro-apoptotic protein Bim (Heman et al. 2005), and that p53 can influence the antitumor activity of certain oncogene-targeted therapeutics (H.G. Wendel et al., in prep.). Nevertheless, these powerful systems have been largely limited to the hematopoietic system.

Combining our previous experience with these novel chimeric mouse model systems, and our desire to easily and accurately model HCCs, we developed and characterized a new mouse model for HCC that is based on the ex vivo manipulation of liver progenitor cells followed by the seeding of these cells into normal recipients. These meth- ods allowed us to rapidly produce in situ liver cancers of defined genetic origin. The generation of the model and its potential applications are the topic of this report.

\section{MATERIALS AND METHODS}

Isolation, culture, and retroviral infection of E-Cadherin-positive liver progenitor cells. Liver cell suspensions from fetal livers of ED12.5-15 C57BL/6 mice (Jackson Laboratories, Rochester, New York), were prepared as described previously (Nitou et al. 2002). Purification of E-Cadherin-positive hepatoblasts from liver suspensions was performed using the MACS $^{\circledR}$ magnetic cell-sorting system (Miltenyi, Auburn, California) in the indirect labeling option together with the rat anti-mouse E-Cadherin (ECCD-1) antibody (Calbiochem, San Diego, California). Columns of the "MS" size were used. Purification was performed by a modification of the protocol recommended by the manufacturer. Briefly, goat anti-rat $\mathrm{IgG}$ microbeads were first incubated with the ECCD-1 antibody for 45 minutes at room temperature with gentle resuspension by pipetting every 5 minutes. 20 $\mu \mathrm{l}$ of immunomagnetic beads were incubated with $4 \mu \mathrm{g}$ of ECCD-1 antibody for later incubation with up to $10^{7}$ positive cells. Complexes of primary and secondary antibody were washed using a MS column according to the manufacturer's instructions. Liver cell suspensions were incubated with the antibody complexes in DMEM 10\% FBS in plastic tubes for 45 minutes at $4^{\circ} \mathrm{C}$. After loading onto MACS MS columns, cells were washed and subsequently eluted in $1 \mathrm{ml}$ of hepatocyte growth medium (Block et al. 1996). The harvest from one embryonic liver was split into two portions and plated in two wells of a 6-well plate that were coated with gelatin (Specialty Media, Phillipsburg, New Jersey) and contained irradiated (30 Gy) NIH3 T3 feeder layers $\left(3 \times 10^{3}\right.$ cells $\left./ \mathrm{cm}^{2}\right)$. Hepatocyte growth medium was supplemented with hepatocyte growth factor (HGF)/SF (40 ng/ml) (Peprotech, Rocky Hill, New Jersey) and epidermal growth factor (EGF) $(20 \mathrm{ng} / \mathrm{ml})$ (Peprotech, Rocky Hill, New Jersey).

After 68 hours, cultured hepatoblasts were transduced with various lentiviral or murine stem cell virus (MSCV)-based retroviral vectors as described previously (Schmitt et al. 2002a), each coexpressing a green fluorescent protein (GFP) reporter. Briefly, retrovirus was produced in Phoenix packaging cells and collected in hepatocyte growth medium. High-titer viral supernatant was passed through a $0.45-\mu \mathrm{m}$ filter and supplemented with polybrene $(2 \mu \mathrm{g} / \mathrm{ml})$, HGF (40 $\mathrm{ng} / \mathrm{ml})$, and EGF $(20 \mathrm{ng} / \mathrm{ml})$. The infection procedure was repeated three times every 8 hours. 24 hours after the last infection, the fraction of GFP-positive cells was assessed by GFP fluorescence microscopy.

Immunofluorescence, immunohistochemistry, and histopathology. Mouse monoclonal antibodies against cytokeratin AE1 (Biocare Medical, Walnut Creek, California) (1:100), OV-6 (R\&D systems, Minneapolis, Minnesota) (1:50), rabbit anti-mouse albumin antiserum (Biogenesis, Poole, United Kingdom) (1:200), rabbit antihuman $\alpha$-fetoprotein (AFP) antiserum (Dako, Glostrup, 
Denmark) (1:200), mouse monoclonal antibodies against cytokeratin 8 (RDI, Concord, Massachusetts) (1:100), or rabbit polyclonal antibodies against GFP (1:200) (Abcam, Cambridge, Massachusetts) were used as primary antibodies. For immunofluorescence of cultured hepatoblasts, cells were either plated on gelatin-coated coverslips/NIH$3 \mathrm{~T} 3$ feeder layers (CK AE1, OV-6, CK8) or on NIH-3T3 feeder layers grown on gelatin-coated plastic wells (AFP, Alb). Cells were fixed with 4\% PFA at room temperature for 15 minutes, permeabilized with $0.2 \%$ Triton, $5 \%$ goat serum in phosphate-buffered saline (PBS) for 5 minutes and subsequently washed with $0.1 \%$ PBS-T, $5 \%$ normal goat serum. Incubation with the primary antibody was performed for 1 hour in $0.1 \%$ PBS-T, $5 \%$ normal goat serum at room temperature. Control samples were incubated with $0.1 \%$ PBS-T, $5 \%$ normal goat serum. Secondary antibodies used were goat anti-mouse Alexa Fluor 594-labeled antibody (Invitrogen, Carlsbad, California) and goat anti-rabbit Alexa Fluor-labeled antibodies (Invitrogen, Carlsbad, California). Anti-GFP immunofluorescence was performed on paraffin-embedded liver sections. Standard Proteinase K antigen-retrieval was used. Detailed protocols for anti-cytokeratin-8 immunohistochemistry on paraffin-embedded liver tumor sections are available upon request. Histopathological evaluation of murine liver carcinomas was done by an experienced liver pathologist (P.F.) using paraffin-embedded liver tumor sections that were stained with hematoxylin/eosin (H\&E) according to standard protocols.

\section{Conditioning of recipient mice and transplantation.} Pathogen-free female C57BL/6 mice (Jackson Laboratories, Rochester, New York), 8-12 weeks of age, weighing 20-30 g were used as recipients for genetically modified liver progenitor cells. Retrorsine and $\mathrm{CCl}_{4}$ stock solutions were prepared as described previously (Guo et al. 2002). Before injection, retrorsine stock solution was diluted 1:5 in sterile PBS. Mice received retrorsine, $70 \mathrm{mg} / \mathrm{kg}$ i.p. This dose was repeated after 7 days and the mice were allowed to recover for 10 days prior to hepatoblast transplantation.

For transplantation of genetically modified hepatoblasts, animals were anesthetized with isolflurane, and a small left subcostal laparotomy was performed. The spleen was retracted and the lower spleen pole looped with a 4-0 vicryl ligation. $2 \times 10^{6}$ cells were injected in 0.2 $\mathrm{ml}$ of medium in the lower pole of the spleen in an injection time of 2 minutes. The lower spleen pole was ligated and resected. The abdomen was sutured using 4-0 vicryl (Ethicon, Norderstedt, Germany). Transplanted cells were allowed to migrate to the recipient liver and engraft the liver. 5 days after intrasplenic cell transplantation, $\mathrm{CCl}_{4}$ was administered in a dose of $0.5 \mathrm{ml} / \mathrm{kg}$ i.p. $\mathrm{CCl}_{4}$ treatment was repeated on d10 and d15 after cell transplantation. Animals were subsequently monitored for tumor formation by abdominal palpation of whole-body fluorescence imaging. For external GFP-tumor imaging, mice were anesthetized with isolflurane, abdominal fur was removed with depilating cream, and animals were monitored by whole-body fluorescence imaging for the retrovirally expressed GFP reporter as described previously (Schmitt et al. 2002a). All procedures were per- formed in accordance with the guidelines of the Institutional Animal Care and Use committee of Cold Spring Harbor Laboratory.

Generation of cell lines and retransplantation. To generate cells from primary HCCs, a tumor nodule of at least $3 \times 3 \mathrm{~mm}$ was removed and diced into small pieces. To obtain a single-cell suspension, minced tumor suspensions were incubated in HEPES-buffered DMEM containing $1000 \mathrm{U} / \mathrm{ml}$ dispase for $30-60$ minutes at $37^{\circ} \mathrm{C}$. The suspension was washed twice in DMEM to remove dispase completely. Before plating, the cell suspension was filtered through a sterile metal sieve to remove clumps. To facilitate the initial attachment, isolated tumor cells were plated on gelatin-coated culture plates.

For retransplantation into recipient mice, $2 \times 10^{6}$ cells were injected in $100 \mu \mathrm{l}$ of medium into the left liver lobe. Briefly, mice were anesthetized as described above. A small ventral midline laparotomy was performed. The left liver lobe was retracted and $2 \times 10^{6}$ cells were injected subcapsularly in $100 \mu \mathrm{l}$ of medium. The injection site was compressed for at least two minutes to prevent bleeding and peritoneal spreading of tumor cells. The intra-abdominal operation field was rinsed with distilled water to osmotically kill accidentally leaked tumor cells. The abdominal wall was closed using 4-0 vicryl suture material (Ethicon, Norderstedt, Germany).

\section{RESULTS}

\section{Isolation and Transduction of Liver Progenitor Cells}

There is increasing evidence that bipotential liver stem cells (oval cells in rodents and hepatic progenitor cells in humans) are the cellular targets for transformation in the development of HCC (Braun et al. 1987; Dumble et al. 2002; Alison and Lovell 2005) and eventually also biliary tract cancer (Tsao and Grisham 1987; Steinberg et al. 1997). Oval cells are bipotential liver stem cells, existing in the healthy adult liver in low numbers around the periportal region. During chronic liver damage, oval cells can proliferate extensively (Thorgeirsson 1996; Dabeva and Shafritz 2003), suggesting that they might be good candidates to target with genetic modifications to model liver cancer. Numerous protocols are available to induce the oval-cell compartment in rodents (for review, see Dabeva and Shafritz 2003), and purification procedures for oval cells have been established (Dumble et al. 2002; Dabeva and Shafritz 2003). However, a major disadvantage of these protocols is that they are based on carcinogen treatment, which may introduce undefined genetic mutations that contribute to tumorigenesis.

Bipotential hepatoblasts are the embryonic counterpart to oval cells from the adult liver (Thorgeirsson 1996; Dabeva and Shafritz 2003). These cells also give rise to cells of both the hepatocytic and bile duct lineage (Fig. 1A) and share almost every marker with oval cells. They exist in large quantities in the murine fetal liver, which also contains hematopoietic cells, endothelial cells, connective tissue cells, and precursors of hepatic stellate cells. Recently, it was reported that hepatoblasts can be purified from the ED12.5 mouse liver using antibodies 
recognizing the extracellular epitopes of E-Cadherin molecules in combination with immunomagnetic beads (Nitou et al. 2002) to a purity of about $98 \%$. These hepatoblasts were capable of spontaneously differentiating into hepatocytes when cocultured with the nonparenchymal fraction of the embryonic liver (Nitou et al. 2002). We therefore reasoned that these embryonic hepatoblasts would serve as an excellent source of liver progenitor cells for further manipulation, and we set out to isolate, culture, and genetically manipulate mouse embryonic hepatoblasts using procedures analogous to our previous efforts in hematopoietic systems.

We isolated E-Cadherin-positive liver progenitor cells using an immunomagnetic-bead-based procedure. After plating, aggregates of hepatoblasts attach in close proximity to the feeder layer cells (Fig. 1B, left). A major limitation to propagating pure hepatoblasts is that the growth and survival of these cells in vitro is limited due to the requirement for hepatoblast-nonparenchymal cell interactions for survival and growth (Nitou et al. 2002). However, we noted that hepatoblasts grown on gelatin-coated culture plates with irradiated NIH-3T3 feeder layers in chemically defined medium (Block et al. 1996) enriched with HGF and EGF are capable of extensive proliferation (Fig. 1B, middle).

Transgenes were introduced into these cells by retrovirus-mediated gene transfer using MSCV-based vectors coexpressing GFP. Using a standard protocol, we typically were able to infect $20-50 \%$ of cells, as assessed by monitoring GFP in the target cell population (Fig. 1B, right). To determine whether these cells retained markers of bipotential liver progenitors, we examined their immunophenotype using several well-established markers (AFP, albumin, CK8, CKAE1, and OV-6) that allow (1) the discrimination of parenchymal from non-parenchymal cells and (2) a classification of the parenchymal cells into progenitor cells, cells of the hepatocytic lineage, and cells of the bile duct lineage.

Immunofluorescence against AFP and albumin (Alb) can be performed on hepatoblasts grown on gelatincoated plastic dishes, thus allowing a quantification of the Alb $\backslash$ AFP-double positive fraction. As expected, the ECadherin-positive liver progenitor cells displayed abundant expression of Alb and AFP, with $>98 \%$ being Alb $\backslash$ AFP-double positive cells. Note that AFP is a marker for bipotential liver progenitor cells that is usually not found in differentiated hepatocytes or bile duct cells. Furthermore, we performed immunofluorescence on the ECadherin-positive liver progenitor cells using a monoclonal antibody directed against the acidic (Type 1) family of cytokeratins. This family consists of cytokeratins 10,14, 15, 16, and 19. Cytokeratins 10, 15, and 16 are mainly found expressed in the skin. Cytokeratins 14 and 19 , however, are known to be markers for bipotential liver progenitor cells (for review, see Thorgeirsson 1996). Positive results were also found in immunofluorescence analyses using antibodies directed against the "liver cytokeratin" cytokeratin 8 and the oval-cell antigen OV-6. The expression levels of CK8, CKAE1, and OV-6 were notably lower than those of Alb and AFP. The re-
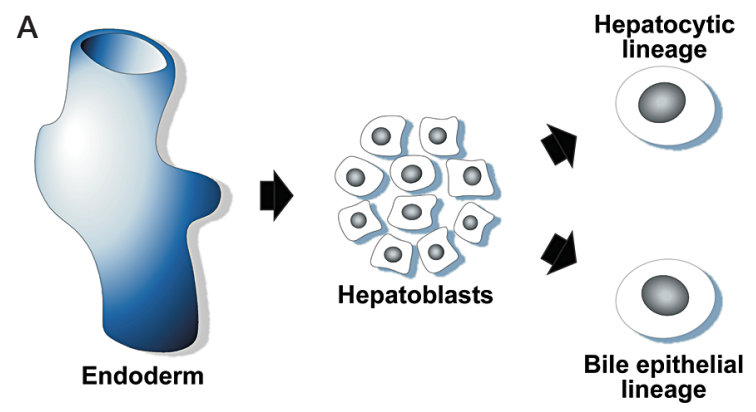

B

brightfield

brightfield
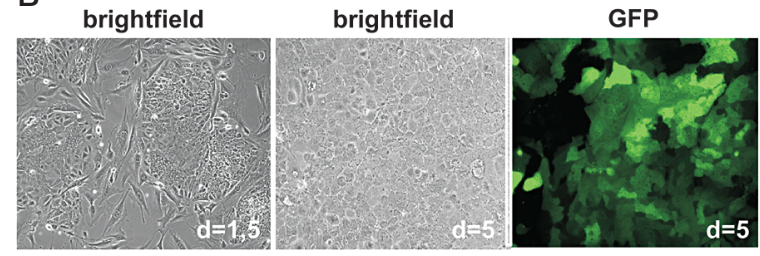

C

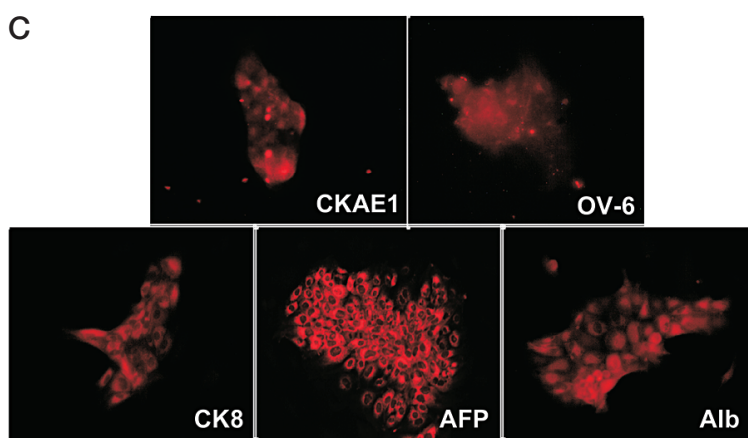

Figure 1. (A) Schematic representation showing the embryonic derivation of hepatic cell lineages. $(B)$ E-Cadherin-positive fetal liver progenitor cells (LPCs, also known as hepatoblasts) can be purified by immunomagnetic beads. E-Cadherin ${ }^{+}$fetal liver progenitor cells are isolated from $\mathrm{ED}=12.5-15$ fetal mouse livers using the MACS $^{\circledR}$ system with indirect labeling. Freshly harvested hepatoblasts are grown in gelatin-coated plates on irradiated NIH-3T3 feeder layers. Islands of LPCs are growing in close proximity to $3 \mathrm{~T} 3$ feeder cells $($ left $)$. E-Cadherin ${ }^{+}$LPCs show an extensive growth potential in vitro when grown on feeder layers in HGF/EGF-enriched hepatocyte growth medium (middle). E-Cadherin ${ }^{+}$LPCs can be infected efficiently with standard retroviral vectors as shown by GFP fluorescence (right). (C) Characterization of the E-Cadherin ${ }^{+}$liver progenitor cells with established hepatoblast/oval cell markers. E-Cadherin ${ }^{+}$LPCs reveal strong positivity for AFP, Alb, and CKAE1; positivity for CK8; and weak positivity for OV-6.

sults of the characterization of the expression pattern of these purified E-Cadherin-positive liver progenitor cells strongly suggest that they are bipotential liver progenitor cells, or hepatoblasts.

\section{Intrahepatic Seeding of Genetically Modified Liver Progenitor Cells}

We next sought to explore whether purified hepatoblasts could be genetically modified and incorporated into the liver. It is known that liver cell transplantation into the spleen of healthy recipient mice leads to an inef- 
ficient repopulation of the host liver $(0.03-0.5 \%$ of the total liver mass) (Gupta et al. 1991; Ponder et al. 1991). Different approaches to facilitate the engraftment of transplanted hepatocytes within the recipient liver have been described. All these approaches share the feature that hepatocytes are transplanted into a predamaged liver which provides a regenerative environment for transplanted cells (Shafritz and Dabeva 2002; Dabeva and Shafritz 2003). Recently, Guo et al. (2002) reported an enhanced repopulation of the mouse liver after pretreatment of the animals with retrorsine. Retrorsine is an alkaloid that exerts a strong and persistent block of native hepatocyte proliferation (Laconi et al. 1999). After hepatocyte transplantation, $\mathrm{CCl}_{4}$ was used to induce a regenerative environment in which transplanted cells can proliferate, while proliferation of recipient cells remains blocked. Using this protocol, Guo et al. (2002) demonstrated repopulation rates of up to $20 \%$ of the liver mass. We reasoned that such procedures in combination with transplantation of genetically modified hepatocytes which exert a proliferation advantage should allow a highly efficient engraftment of transplanted cells.

A schematic representation of our approach is shown in Figure 2A. E-Cadherin-positive bipotential liver progenitor cells are purified and grown in primary culture. Cells are infected with retroviral constructs, expressing oncogenes or short hairpin RNAs (shRNAs) directed against tumor suppressor genes. After infection, $2 \times 10^{6}$ liver progenitor cells are transplanted into the spleens of recipient mice that were conditioned with retrorsine. One week after intrasplenic injection of the cells, a cycle of three $\mathrm{CCl}_{4}$ treatments is started. The timeline of the retrorsine pretreatment and subsequent $\mathrm{CCl}_{4}$ treatment is shown in Figure $2 \mathrm{~B}$. One week after intrasplenic injection of GFPtagged liver progenitor cells, livers were harvested and processed for immunofluorescence with an antibody directed against GFP. We found that approximately $1 \%$ of the whole liver consisted of the "seeded," GFP-positive liver progenitor cells. Interestingly, on H\&E sections these transplanted cells are indistinguishable from the surrounding normal hepatocytes (Fig. 2C).

\section{Generation of Liver Cancers In Situ}

Our next goal was to generate primary liver carcinomas with defined genetic lesions previously linked to the development of human HCC. For example, mutations in $\mathrm{p} 53, \mathrm{Rb}$, and IGFR1 are frequently found in HCC (Murakami et al. 1991; Kim et al. 1996), and the oncogenes c-myc and cyclin D1 are frequently found to be overexpressed (Peng et al. 1993; Nishida et al. 1994). To generate primary liver carcinomas of the genetic compound lesion "p $53^{-1-}$;c-myc," we purified hepatoblasts from p53-deficient embryonic mouse livers. These cells were transduced with an MSCV-based retroviral vector coexpressing c-myc with a GFP reporter and transplanted into the spleen of retrorsine-pretreated recipient mice as described above. Animals were continuously monitored for general health and tumor formation by abdominal palpation and external GFP fluorescence imaging.
Figure 2D shows a tumor-bearing mouse with a swollen abdomen due to ascites (digital picture, right panel). The tumor is detectable by whole-body fluorescence imaging (left panel). The asterisks indicate remnants of GFP-positive transplanted hepatocytes in the spleen. GFP imaging of the explanted liver (lower panel, left) shows an advanced liver carcinoma filling a whole liver lobe. It is apparent that the external GFP signal is not proportional to the real tumor size, because about $70 \%$ of the GFP signal is absorbed by the ribcage/abdominal wall. Presumably, more sensitive imaging techniques, such as bioluminescence using a luciferase reporter, would enable the detection of small liver tumors and precise assessment of partial responses in treatment studies.

We also generated in situ liver carcinomas by seeding genetically manipulated adult liver cells (no separation of adult hepatocytes from liver progenitor cells) obtained by collagenase perfusion from livers of $\mathrm{p} 53$-deficient mice (data not shown). Because the infection efficiency of differentiated hepatocytes with standard retroviral vectors is much lower compared to hepatoblasts (data not shown), we used lentiviral vectors in this setting. Infection of p53deficient adult liver cells with a lentivirus expressing an HA-tagged form of c-myc (Hemann et al. 2005) and seeding of these cells into the livers of conditioned recipient mice gave rise to in situ liver carcinomas with lower penetrance than the hepatoblast cultures. We also found that tumorigenicity of $\mathrm{p} 53^{-/-}$embryonic liver cells isolated from the late-stage mouse embryo (ED16-18) and infected with a c-myc-expressing retrovirus is significantly lower than tumorigenicity of cells of the same genotype that were harvested around ED12.5 (data not shown). Interestingly, embryonic hepatocytes that were harvested from ED18 p53 ${ }^{-/-}$embryonic mouse livers and infected with a retrovirus encoding c-myc are only immortalized but not transformed $(0 / 8$ tumors subcutaneously on nude mice, $0 / 12$ tumors in situ).

We next established a protocol for the culture and expansion of hepatoma cells from primary murine HCCs of a defined genetic origin (see Materials and Methods for details). In most instances, this procedure allowed the establishment of a stable population of cultured hepatoma cells. After expansion, the cells could be directly injected into the liver of recipient mice. Figure $2 \mathrm{E}$ shows an explanted liver several weeks after direct infiltration of $2 x$ $10^{6}$ " $\mathrm{p} 53^{-/-}$; c-myc" hepatoma cells into the left liver lobe. The tumor is readily visualized by GFP imaging as shown in the left panel of the figure. The ability to generate multiple tumors of exactly the same genetic background should enable side-by-side comparisons of different treatment regimens in tumors of the same genetic background.

\section{Suppression of Gene Expression in Murine HCC Using Stable RNA Interference}

Historically, mouse models of human liver cancer have involved the germ-line manipulation of either oncogenes or tumor suppressor genes. In the case of tumor suppressor genes, one either constitutively deletes the tumor suppressor gene or produces a conditional 
A

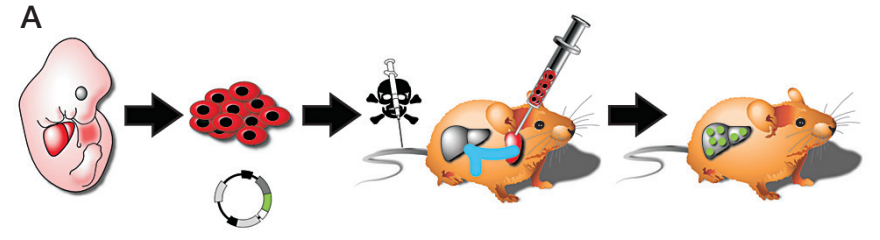

B

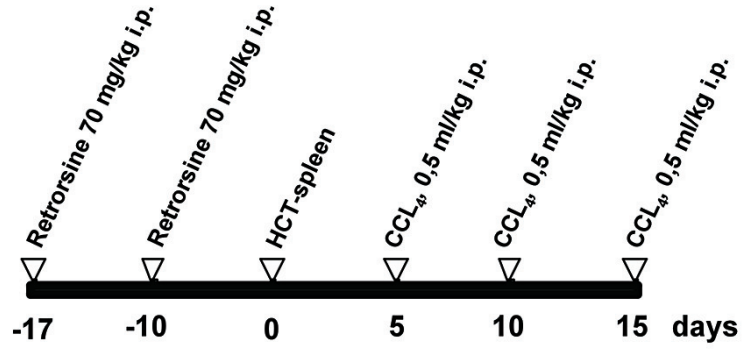

C

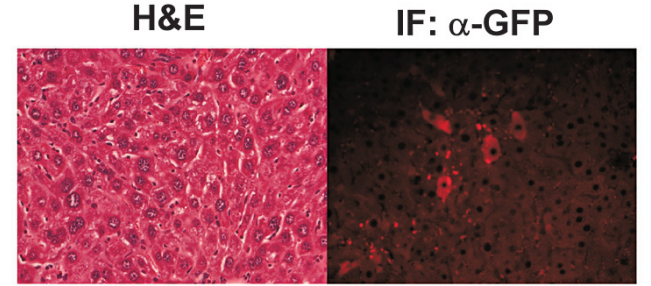

D

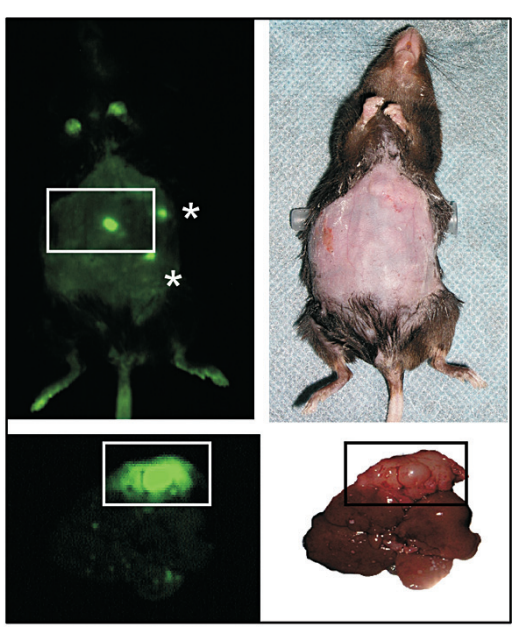

$E$

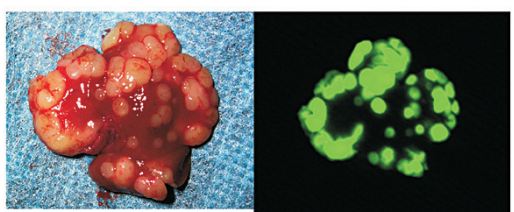

Figure 2. Generation of genetically defined in situ liver cancers. (A) Technical outline. E-Cadherin-positive fetal liver progenitor cells (hepatoblasts) are purified and cultured as described above. Using the murine stem cell virus (MSCV) optimized to drive longterm gene expression in vivo, the cells are infected with oncogenes and/or shRNAs directed against tumor suppressor genes. After infection and expansion, the cells are transplanted into the spleens of conditioned recipient mice. Because all retroviral vectors used for infections are GFP-tagged, development of in situ HCCs can be monitored by external GFP imaging. (B) Schematic representation of the timeline of the approach. Mice undergo two pretreatments with the liver cell cycle inhibitor retrorsine. After transplantation of the cells into the spleen, the transplanted hepatoblasts are selectively expanded by $\mathrm{CCl}_{4}$ treatment of the mice. $(C) \mathrm{GFP}^{+}$ transplanted hepatoblasts can be detected in the recipient liver by anti-GFP immunofluorescence (right). H\&E staining of an adjacent section of the liver (left). (D) External GFP imaging of a p53; c-myc tumor-bearing mouse (left). The GFP-positive spot in the square represents the intrahepatic tumor mass. The two additional spots (marked by asterisks) represent transplanted cells residing in the spleen after transplantation. Mice with advanced intrahepatic tumor growth present with swollen, ascites-containing abdomen allowing detection of tumor burden by palpation. GFP imaging of the explanted liver (bottom panel) reveals an advanced intrahepatic tumor, filling out a whole liver lobe. $(E)$ Primary liver tumors can be outgrown in culture and retransplanted in situ into recipient mice. Shown is a tumor that was retransplanted by direct liver injection of $2 \times 10^{6}$ tumor cells. Note the extensive intrahepatic metastasis of the transplanted cells.

"knockout" animal where the gene is flanked by loxP sites such that the tumor suppressor gene can be inactivated in specific settings using cre recombinase. Although extremely powerful, these approaches can be extremely time- and cost-intensive and, in the case of constitutive knockout strains, are subject to developmental compensation that can mask aspects of the null phenotype (Sage et al. 2003). However, over the last several years, RNA interference (RNAi) has emerged as a complementary and effective tool to homologous recombination to study the consequences of gene suppression in cultured mammalian cells and in mice (Hemann et al. 2003; Hannon and Rossi 2004; Dickins et al. 2005). Furthermore, improved reagents for stable RNAi have been reported where the silencing triggers mimic a natural microRNA primary transcript, and each target sequence has been selected on the basis of thermodynamic criteria for optimal shRNA performance (Dickins et al. 2005; Silva et al. 2005). We were therefore interested in evalu- ating whether stable RNAi technology could be harnessed to create dominantly acting inhibitors of tumor suppressor function in a chimeric (genetic mosaic) model of hepatocarcinogenesis.

To test the feasibility of such an approach, we sought to generate in situ liver carcinomas by genetic manipulation of bipotential liver progenitor cells with synthetic microRNAs directed against p53 in combination with different oncogenes. The predicted folding of the primary microRNA is shown in Figure 3A. The red bars indicate the predicted mature small interfering RNA. Figure $3 \mathrm{~B}$ shows in situ liver carcinomas derived either from p53-deficient liver progenitor cells (LPCs) overexpressing oncogenic Ras or from wild-type LPCs that were double infected with a microRNA-based sh-construct directed against p53 (MLS.1224), together with a vector expressing oncogenic Ras. The infected cell populations were seeded into the liver or injected subcutaneously into an immunocompromised mouse. 
GFP fluorescence revealed that liver carcinomas developed rapidly in the livers of transplanted recipient mice $(<3$ weeks), irrespective of whether they were derived from $\mathrm{p} 53^{-1-}$ hepatoblasts or wild-type hepatoblasts expressing sh-p53. Indeed, tumors arising from both configurations displayed a highly aggressive and disseminated growth pattern in the liver (Fig. 3B). Tumors also arose rapidly in the subcutaneous setting, where they could be readily monitored using standard caliper measurements. The growth rates of liver carcinomas derived from " $\mathrm{p} 53^{-1-}$;H-RasV12" tumors show no significant difference from "shp53;H-RasV12" tumors (Fig. 3C), implying that, in this setting, RNAi was as effective at promoting tumorigenesis as a null allele. Representative GFP images from both groups are shown along with their respective tumor growth curves. To our knowledge, this is the first demonstration of the generation of in situ carcinomas by stable RNAi.

\section{Murine Liver Cancers Resemble Primary Human Liver Cancer}

For a mouse model to have maximal utility, it is important to understand the extent to which it recapitulates the histopathology of the corresponding human disease. In humans, the histopathological appearance of HCC is extremely varied, with several distinct growth patterns. These different histopathological patterns have no clinical or prognostic significance in humans with the exception of "fibrolamellar carcinoma" (a rare variant of HCC that can respond to chemoradiation therapy). To validate that the tumors of our mouse model resemble the human pathology, we analyzed a large panel of tumors based on the compound genetic lesion "p53-/-;c-myc" for the appearance of different histopathological subtypes. H\&Estained sections from paraffin-embedded tissues were examined by an experienced liver pathologist (P.F.). More than $80 \%$ of the examined "c-myc;p53 $53^{-1}$ HCCs" were classified as moderately well to poorly differentiated HCCs with a mostly solid, sometimes mixed solid/trabecular growth pattern (Fig. 4). All tumors were additionally stained with cytokeratin 8 to confirm the affiliation with the liver lineage (Thorgeirsson 1996). A much smaller proportion of tumors $(<20 \%)$ represented histological growth patterns resembling human trabecular HCC or pseudoglandular HCC (Fig. 4). Thus, tumors arising through liver seeding of genetically altered liver progenitor cells show a remarkable similarity to human liver cancers.

\section{DISCUSSION}

\section{A New Mouse Model of Liver Cancer}

Although HCC is one of the most frequent neoplasms worldwide, there is an insufficient genetic and biological understanding of the disease. Based on our technological foundations from mouse models of blood-related malignancies (Schmitt et al. 2002a,b; Hemann et al. 2005), we sought to develop a chimeric new orthotopic, genetically tractable mouse model for HCC that would allow a more

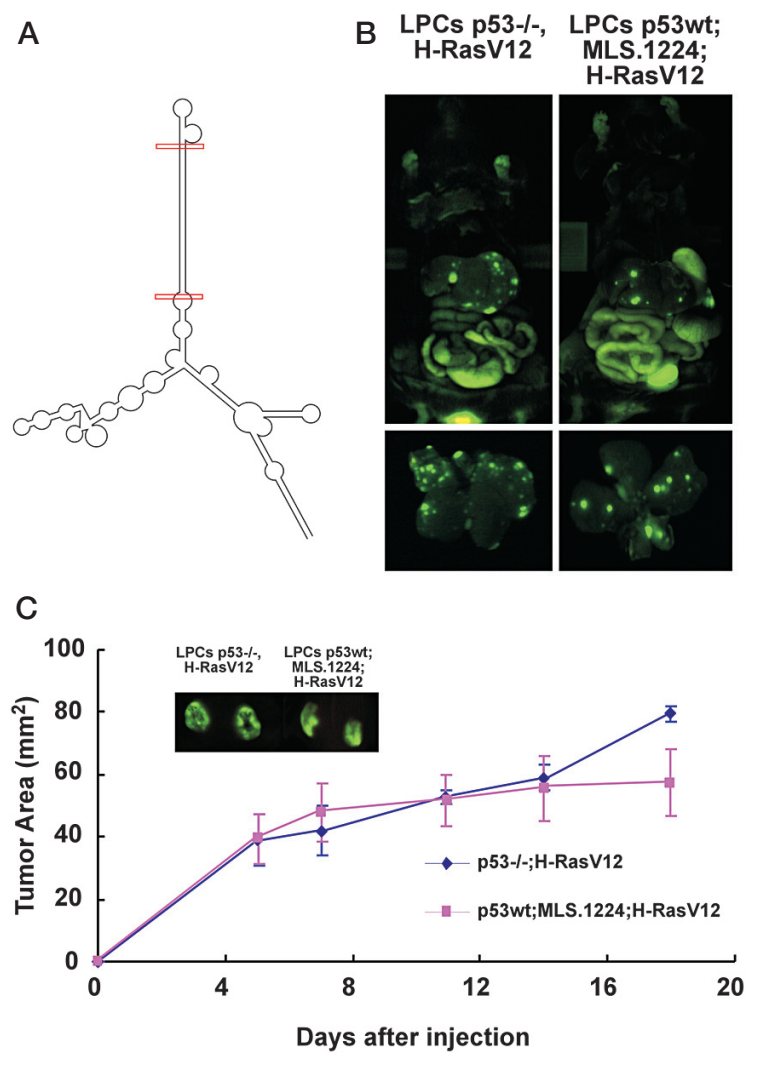

Figure 3. Second-generation, microRNA-based shRNA expression vectors can be used to model hepatocarcinogenesis in vivo. $(A)$ Predicted folding for the pri-microRNA. The predicted mature siRNA duplex is labeled with red bars. $(B)$ Liver carcinomas were generated either by infecting $\mathrm{p} 53^{-1}$ liver progenitor cells (LPCs) with oncogenic Ras or by coinfecting wild-type LPCs with a microRNA-based shRNA construct directed against p53 (MLS.1224) together with oncogenic Ras. (C) To facilitate the exact measurement of tumor growth, "p53 $3^{-/} ; \mathrm{H}-\mathrm{RasV} 12$ " and "sh-p53;H-RasV12" liver carcinomas were also grown subcutaneously on NCR nu/nu mice. Shown are representative external GFP imagings of the subcutaneously grown tumors and tumor growth curves for each group. Growth rates of "p $53^{-/} ; \mathrm{H}-\mathrm{RasV} 12$ " and "sh-p $53 ; \mathrm{H}-$ RasV12" liver carcinomas were assessed by caliper measurements ( $n=6$ in each group).

effective analysis of this disease. Our approach allows genetic manipulation of bipotential liver progenitor cells ex vivo with subsequent seeding of these cells into the livers of conditioned recipient mice. Following the introduction of appropriate genetic combinations, this procedure results in the development of in situ liver tumors that resemble human $\mathrm{HCC}$.

Our model is based on the targeting of liver progenitor cells with initiating oncogenic lesions. It is an emerging concept that tissue-specific stem or progenitor cells can be the target for transformation in different types of human cancer (Smalley and Ashworth 2003; Al Hajj and Clarke 2004; Baik et al. 2004; Tai et al. 2005). For liver cancer, it remains under debate whether carcinomas are derived from differentiated hepatocytes or from liver progenitor cells. However, there is increasing evidence that bipoten- 


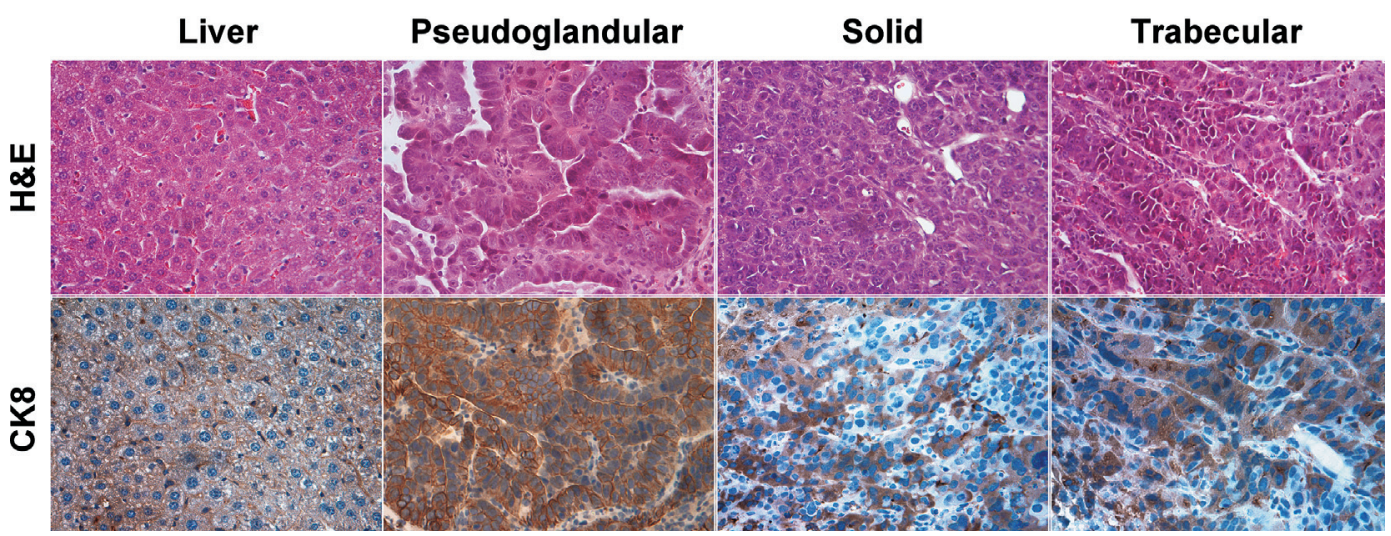

Figure 4. Mouse HCCs derived from E-Cadherin ${ }^{+}$liver progenitor cells reveal histopathological subtypes of human HCC. Three common variegations of human HCCs (solid, trabecular, and pseudoglandular growth pattern) are lined up with corresponding mouse HCCs derived from genetically modified hepatoblasts. All shown mouse HCCs are derived from p53 $3^{-/}$hepatoblasts overexpressing c-myc. Mouse HCCs were stained for cytokeratin 8 as a typical liver cytokeratin.

tial liver stem cells, so-called oval cells, are the cellular targets for transformation in the development of HCC (Braun et al. 1987; Dumble et al. 2002; Alison and Lovell 2005). In our model, bipotential liver progenitor cells from the fetal mouse liver are the target for transformation in the development of liver carcinomas. Tumors derived from genetically modified hepatoblasts closely resemble the histopathology of human liver cancer, with respect to both their general pathology and underlying genetics.

Genes were introduced into liver progenitor cells by retroviral transduction, and infected populations were introduced into livers of "conditioned" recipient mice under circumstances that facilitiate engraftment of these cells into the liver. Of note, direct delivery of concentrated lentivirus overexpressing c-myc into the livers of p53-deficient mice gave no liver tumors in an observation period of 1 year (L. Zender and S.W. Lowe, unpubl.). This observation suggests that infiltration of lentivirus into the adult mouse liver predominantly infected differentiated hepatocytes, providing further support for a stem cell theory of hepatocarcinogenesis. Our observations are in accordance with a recent study of Lewis et al. (2005), who demonstrated that c-myc delivered in RCAS vectors into the livers of Alb-TVA p53-deficient mice was not capable of forming liver tumors. The fact that these investigators were able to generate liver carcinomas by delivering the mouse polyomavirus middle-T antigen (PyMT) in RCAS vectors into p53-deficient Alb-TVA may be due to the fact that PyMT-induced transformation is more efficient than c-myc-induced transformation, and a small subfraction of targeted bipotential liver progenitor cells (oval cells) is sufficient to give rise to tumors.

Our approach to generate genetically defined liver carcinomas has several advantages compared to classic transgenic approaches. First, it is a very time- and costeffective way to produce high numbers of genetically defined HCCs. Second, mosaics are generated, such that genetically modified cells are surrounded by otherwise normal counterparts, allowing the development of tumors that resembles the pathogenesis of human HCC. Third, all experiments can be conducted in a single genetic background using the same constitutively active promoter, which facilitates the comparison of results between different investigators. Fourth, because the retroviruses used to produce the tumors can have imaging reporters, the resulting tumors can be detected and monitored using inexpensive imaging technologies, such as whole-body fluorescence imaging. Fifth, primary liver carcinomas of defined genetic origin can be grown ex vivo and retransplanted directly into the livers of recipient mice, a property that facilitates the use of this model in preclinical trials to test new treatment regimens against HCC. Finally, tumor-bearing mice can be generated without the use of germ-line methods, thereby circumventing some of the constraints of the "Oncomouse" patent.

\section{Probing HCC Genetics and Biology}

Despite the fact that HCC has a huge impact on human health, relatively little is known about the molecular mechanisms of hepatocarcinogenesis. To gain further insights into liver cancer genetics and to define possible therapeutic targets against liver cancer, we applied a high-resolution genome-wide scanning technology to murine liver carcinomas that were initiated by two defined hits. We demonstrate that a mouse liver tumor that was primarily driven by oncogenic ras acquired a focal genomic amplification containing c-myc, a gene highly established as associated with human liver cancer (data not shown).

These results underscore the potential of our approach to identify new candidate genes for hepatocarcinogenesis. Indeed, in a parallel study (L. Zender et al., in prep.), we have taken advantage of our model to identify, validate, and characterize a novel oncogene linked to human HCC. Specifically, we conducted ROMA on a series of murine HCCs arising in the presence of Myc, Akt, or oncogenic Ras, and noted that the Myc-expressing HCCs (and not those expressing Akt or Ras) frequently harbored an amplification on mouse chromosome 9. Inter- 
estingly, by analyzing a series of human HCCs, we found a corresponding amplification on chromosome 11, which is syntenic to mouse chromosome 9 and contained the same set of genes. Through further cross-species analysis and expression studies, we narrowed down one genethe cellular inhibitor of apoptosis gene cIAP1 - as the most likely "driver" gene in this amplicon.

One powerful aspect of this system is that genes identified as altered in the tumors can be rapidly examined for oncogenic potential in the mouse using the precise genetic configuration in which the spontaneous lesion arose. Thus, by returning to the mouse, we demonstrated that enforced expression of cIAP1 in the genetic context in which the amplification occurred ( $\left.553^{-1} ; \mathrm{c}-\mathrm{Myc}\right)$ accelerated tumorigenesis and, conversely, suppression of cIAP1 in cells harboring the amplicon reduced tumor growth. These effects were highly genotype-specificthus, cIAP1 had no impact on promoting tumors initiated with Akt or Ras, and shRNAs targeting cIAP did not delay the growth of tumors that did not contain the cIAP amplicon. In addition to validating cIAP1 as a bona fide oncogene, these studies define an integrative approach to cancer genetics and biology that may facilitate the annotation of the cancer genome (for more discussion, see L. Zender et al., in prep.).

In addition to identifying new genes involved in HCC using genome-scanning approaches followed by "reverse genetics," our model is amenable to forward genetics approaches using new, low-complexity cDNA or RNAi libraries to identify new oncogenes and tumor suppressor genes, respectively. In principle, one can envision introducing defined "pools" of cDNAs or shRNAs into hepatoblasts from a "sensitized" genetic background followed by transplantation into the livers of recipient mice. In principle, if a given pool harbors an oncogenic cDNA or shRNA, tumors should arise more rapidly than controls. Here, we provide a proof-of-principle experiment that microRNA-based shRNAs can be used to generate in situ liver carcinomas. This HCC mouse model should prove to be a valuable tool to further explore molecular mechanisms of hepatocarcinogenesis and to help define new molecular targets.

\section{A Preclinical Mouse Model for Testing New Therapies}

Human liver cancer currently represents the third leading cause of cancer deaths; in part, because no effective chemotherapeutic regimens are currently available. Thus, a major effort of HCC research is to develop the molecular and modeling infrastructure to identify new drug targets and test their potential efficacy. As mentioned above, our chimeric mouse model of HCC has many features that should enable it to provide new insights into the molecular genetics of HCC. In addition, as shown in this study, all of the liver tumors we produce can be monitored by GFP imaging, and most can be propagated in vitro or by transplanting into the livers of syngeneic recipients. Although more sensitive imaging techniques such as bioluminescence should improve monitoring ca- pabilities, the availability of defined tumors and simple imaging modalities should enable relatively highthroughput preclinical studies using new drugs or drug combinations on HCC. Such approaches, particularly with new molecularly targeted therapies, may identify new treatment regimens that may be effective against liver cancers with a defined set of underlying genetic lesions.

\section{ACKNOWLEDGMENTS}

We thank Amy Brady, Christine Rosenthal, Lisa Bianco, and Maria S. Jiao for excellent technical assistance, and Dr. Eva Hernando for assistance with histology. We also acknowledge Michael Wigler, Lakshmi Muthuswamy, and other members of the Wigler group for providing the bioinformatics required for the mouse ROMA chip and ROMA data analysis, and the members of the Lowe lab for constructive criticism and discussions throughout the course of this work. L.Z. was supported by the German Research foundation (Emmy Noether Programme). This work was also supported by a grant from the Alan Seligson foundation, and by grants CA13106, CA87497, and CA105388 from the National Institutes of Health. S.W.L. and G.J.H. are Howard Hughes Medical Institute investigators.

\section{REFERENCES}

Al Hajj M. and Clarke M.F. 2004. Self-renewal and solid tumor stem cells. Oncogene 23: 7274.

Alison M.R. and Lovell M.J. 2005. Liver cancer: The role of stem cells. Cell Prolif. 38: 407.

Baik I., Becker P.S., DeVito W.J., Lagiou P., Ballen K., Quesenberry P.J., and Hsieh C.C. 2004. Stem cells and prenatal origin of breast cancer. Cancer Causes Control 15: 517.

Block G.D., Locker J., Bowen W.C., Petersen B.E., Katyal S., Strom S.C., Riley T., Howard T.A., and Michalopoulos G.K. 1996. Population expansion, clonal growth, and specific differentiation patterns in primary cultures of hepatocytes induced by HGF/SF, EGF and TGF alpha in a chemically defined (HGM) medium. J. Cell Biol. 132: 1133.

Braun L., Goyette M., Yaswen P., Thompson N.L., and Fausto N. 1987. Growth in culture and tumorigenicity after transfection with the ras oncogene of liver epithelial cells from carcinogen-treated rats. Cancer Res. 47: 4116.

Bruix J., Boix L., Sala M., and Llovet J.M. 2004. Focus on hepatocellular carcinoma. Cancer Cell 5: 215.

Buendia M.A. 2000. Genetics of hepatocellular carcinoma. Semin. Cancer Biol. 10: 185.

Dabeva M.D. and Shafritz D.A. 2003. Hepatic stem cells and liver repopulation. Semin. Liver Dis. 23: 349.

Deane N.G., Parker M.A., Aramandla R., Diehl L., Lee W.J., Washington M.K., Nanney L.B., Shyr Y., and Beauchamp R.D. 2001. Hepatocellular carcinoma results from chronic cyclin D1 overexpression in transgenic mice. Cancer Res. 61: 5389.

Dickins R.A., Hemann M.T., Zilfou J.T., Simpson D.R., Ibarra I., Hannon G.J., and Lowe S.W. 2005. Probing tumor phenotypes using stable and regulated synthetic microRNA precursors. Nat. Genet. 37: 1289.

Dumble M.L., Croager E.J., Yeoh G.C., and Quail E.A. 2002. Generation and characterization of p53 null transformed hepatic progenitor cells: Oval cells give rise to hepatocellular carcinoma. Carcinogenesis 23: 435.

Guo D., Fu T., Nelson J.A., Superina R.A., and Soriano H.E. 2002. Liver repopulation after cell transplantation in mice treated with retrorsine and carbon tetrachloride. Transplantation 73: 1818 . 
Gupta S., Aragona E., Vemuru R.P., Bhargava K.K., Burk R.D., and Chowdhury J.R. 1991. Permanent engraftment and function of hepatocytes delivered to the liver: Implications for gene therapy and liver repopulation. Hepatology 14: 144.

Hannon G.J. and Rossi J.J. 2004. Unlocking the potential of the human genome with RNA interference. Nature 431: 371.

Harada N., Oshima H., Katoh M., Tamai Y., Oshima M., and Taketo M.M. 2004. Hepatocarcinogenesis in mice with betacatenin and Ha-ras gene mutations. Cancer Res. 64: 48.

Hemann M.T., Fridman J.S., Zilfou J.T., Hernando E., Paddison P.J., Cordon-Cardo C., Hannon G.J., and Lowe S.W. 2003. An epi-allelic series of p53 hypomorphs created by stable RNAi produces distinct tumor phenotypes in vivo. Nat. Genet. 33: 396.

Hemann M.T., Bric A., Teruya-Feldstein J., Herbst A., Nilsson J.A., Cordon-Cardo C., Cleveland J.L., Tansey W.P., and Lowe S.W. 2005. Evasion of the p53 tumour surveillance network by tumour-derived MYC mutants. Nature 436: 807.

Huo T.I., Wang X.W., Forgues M., Wu C.G., Spillare E.A., Giannini C., Brechot C., and Harris C.C. 2001. Hepatitis B virus $\mathrm{X}$ mutants derived from human hepatocellular carcinoma retain the ability to abrogate p53-induced apoptosis. Oncogene 20: 3620 .

Jhappan C., Stahle C., Harkins R.N., Fausto N., Smith G.H., and Merlino G.T. 1990. TGF alpha overexpression in transgenic mice induces liver neoplasia and abnormal development of the mammary gland and pancreas. Cell 61: 1137.

Kim S.O., Park J.G., and Lee Y.I. 1996. Increased expression of the insulin-like growth factor I (IGF-I) receptor gene in hepatocellular carcinoma cell lines: Implications of IGF-I receptor gene activation by hepatitis B virus $\mathrm{X}$ gene product. Cancer Res. 56: 3831 .

Kusano N., Shiraishi K., Kubo K., Oga A., Okita K., and Sasaki K. 1999. Genetic aberrations detected by comparative genomic hybridization in hepatocellular carcinomas: Their relationship to clinicopathological features. Hepatology 29: 1858.

Laconi S., Curreli F., Diana S., Pasciu D., De Filippo G., Sarma D.S., Pani P., and Laconi E. 1999. Liver regeneration in response to partial hepatectomy in rats treated with retrorsine: A kinetic study. J. Hepatol. 31: 1069.

Lewis B.C., Klimstra D.S., Socci N.D., Xu S., Koutcher J.A., and Varmus H.E. 2005. The absence of p53 promotes metastasis in a novel somatic mouse model for hepatocellular carcinoma. Mol. Cell. Biol. 25: 1228.

Llovet J.M., Burroughs A., and Bruix J. 2003. Hepatocellular carcinoma. Lancet 362: 1907.

Lucito R., Healy J., Alexander J., Reiner A., Esposito D., Chi M., Rodgers L., Brady A., Sebat J., Troge J., West J.A., Rostan S., Nguyen K.C., Powers S., Ye K.Q., Olshen A., Venkatraman E., Norton L., and Wigler M. 2003. Representational oligonucleotide microarray analysis: A high-resolution method to detect genome copy number variation. Genome Res. 13: 2291.

Manickan E., Satoi J., Wang T.C., and Liang T.J. 2001. Conditional liver-specific expression of simian virus $40 \mathrm{~T}$ antigen leads to regulatable development of hepatic neoplasm in transgenic mice. J. Biol. Chem. 276: 13989.

Murakami H., Sanderson N.D., Nagy P., Marino P.A., Merlino G., and Thorgeirsson S.S. 1993. Transgenic mouse model for synergistic effects of nuclear oncogenes and growth factors in tumorigenesis: Interaction of c-myc and transforming growth factor alpha in hepatic oncogenesis. Cancer Res. 53: 1719.

Murakami Y., Hayashi K., Hirohashi S., and Sekiya T. 1991. Aberrations of the tumor suppressor p53 and retinoblastoma genes in human hepatocellular carcinomas. Cancer Res. 51: 5520 .

Nhieu J.T., Renard C.A., Wei Y., Cherqui D., Zafrani E.S., and Buendia M.A. 1999. Nuclear accumulation of mutated betacatenin in hepatocellular carcinoma is associated with increased cell proliferation. Am. J. Pathol. 155: 703.

Niketeghad F., Decker H.J., Caselmann W.H., Lund P., Geissler F., Dienes H.P., and Schirmacher P. 2001. Frequent genomic imbalances suggest commonly altered tumour genes in hu- man hepatocarcinogenesis. Br. J. Cancer 85: 697.

Nishida N., Fukuda Y., Komeda T., Kita R., Sando T., Furukawa M., Amenomori M., Shibagaki I., Nakao K., and Ikenaga M., et al. 1994. Amplification and overexpression of the cyclin D1 gene in aggressive human hepatocellular carcinoma. Cancer Res. 54: 3107.

Nitou M., Sugiyama Y., Ishikawa K., and Shiojiri N. 2002. Purification of fetal mouse hepatoblasts by magnetic beads coated with monoclonal anti-e-cadherin antibodies and their in vitro culture. Exp. Cell Res. 279: 330.

Parkin D.M., Bray F., Ferlay J., and Pisani P. 2001. Estimating the world cancer burden: Globocan 2000. Int. J. Cancer 94: 153.

Pear W.S., Miller J.P., Xu L., Pui J.C., Soffer B., Quackenbush R.C., Pendergast A.M., Bronson R., Aster J.C., Scott M.L., and Baltimore D. 1998. Efficient and rapid induction of a chronic myelogenous leukemia-like myeloproliferative disease in mice receiving P210 bcr/abl-transduced bone marrow. Blood 92: 3780.

Peng S.Y., Lai P.L., and Hsu H.C. 1993. Amplification of the cmyc gene in human hepatocellular carcinoma: Biologic significance. J. Formos. Med. Assoc. 92: 866.

Ponder K.P., Gupta S., Leland F., Darlington G., Finegold M., DeMayo J., Ledley F.D., Chowdhury J.R., and Woo S.L. 1991. Mouse hepatocytes migrate to liver parenchyma and function indefinitely after intrasplenic transplantation. Proc. Natl. Acad. Sci. 88: 1217.

Ray R.B., Steele R., Meyer K., and Ray R. 1997. Transcriptional repression of $\mathrm{p} 53$ promoter by hepatitis $\mathrm{C}$ virus core protein. J. Biol. Chem. 272: 10983.

Sage J., Miller A.L., Perez-Mancera P.A., Wysocki J.M., and Jacks T. 2003. Acute mutation of retinoblastoma gene function is sufficient for cell cycle re-entry. Nature 424: 223.

Sandgren E.P., Quaife C.J., Pinkert C.A., Palmiter R.D., and Brinster R.L. 1989. Oncogene-induced liver neoplasia in transgenic mice. Oncogene 4: 715 .

Schmitt C.A., Fridman J.S., Yang M., Baranov E., Hoffman R.M., and Lowe S.W. 2002a. Dissecting p53 tumor suppressor functions in vivo. Cancer Cell 1: 289.

Schmitt C.A., Fridman J.S., Yang M., Lee S., Baranov E., Hoffman R.M., and Lowe S.W. 2002b. A senescence program controlled by p53 and p16INK4a contributes to the outcome of cancer therapy. Cell 109: 335.

Shachaf C.M., Kopelman A.M., Arvanitis C., Karlsson A., Beer S., Mandl S., Bachmann M.H., Borowsky A.D., Ruebner B., Cardiff R.D., Yang Q., Bishop J.M., Contag C.H., and Felsher D.W. 2004. MYC inactivation uncovers pluripotent differentiation and tumour dormancy in hepatocellular cancer. Nature 431: 1112.

Shafritz D.A. and Dabeva M.D. 2002. Liver stem cells and model systems for liver repopulation. J. Hepatol. 36: 552.

Silva J.M., Li M.Z., Chang K., Ge W., Golding M.C., Rickles R.J., Siolas D., Hu G., Paddison P.J., Schlabach M.R., Sheth N., Bradshaw J., Burchard J., Kulkarni A., Cavet G., Sachidanandam R., McCombie W.R., Cleary M.A., Elledge S.J., and Hannon G.J. 2005. Second-generation shRNA libraries covering the mouse and human genomes. Nat. Genet. 37: 1281.

Smalley M. and Ashworth A. 2003. Stem cells and breast cancer: A field in transit. Nat. Rev. Cancer 3: 832.

Steinberg P., Frank H., Odenthal M., Dienes H.P., and Seidel A. 1997. Role of the Ha-ras gene in the malignant transformation of rat liver oval cells. Int. J. Cancer 71: 680.

Tai M.H., Chang C.C., Kiupel M., Webster J.D., Olson L.K., and Trosko J.E. 2005. Oct4 expression in adult human stem cells: Evidence in support of the stem cell theory of carcinogenesis. Carcinogenesis 26: 495.

Thorgeirsson S.S. 1996. Hepatic stem cells in liver regeneration. FASEB J. 10: 1249.

Tsao M.S. and Grisham J.W. 1987. Hepatocarcinomas, cholangiocarcinomas, and hepatoblastomas produced by chemically transformed cultured rat liver epithelial cells. A light- and electron-microscopic analysis. Am. J. Pathol. 127: 168.

Ueda H., Ullrich S.J., Gangemi J.D., Kappel C.A., Ngo L., Feitelson M.A., and Jay G. 1995. Functional inactivation but not 


\section{GENETICALLY DEFINED LIVER CARCINOMAS}

structural mutation of p53 causes liver cancer. Nat. Genet. 9: 41.

Verna L., Whysner J., and Williams G.M. 1996a. 2-Acetylaminofluorene mechanistic data and risk assessment: DNA reactivity, enhanced cell proliferation and tumor initiation. Pharmacol. Ther. 71: 83.

. 1996b. N-nitrosodiethylamine mechanistic data and risk assessment: Bioactivation, DNA-adduct formation, mutagenicity, and tumor initiation. Pharmacol. Ther. 71: 57.

Wang R., Ferrell L.D., Faouzi S., Maher J.J., and Bishop J.M. 2001. Activation of the Met receptor by cell attachment induces and sustains hepatocellular carcinomas in transgenic mice. J. Cell Biol. 153: 1023.
Wang X.W., Forrester K., Yeh H., Feitelson M.A., Gu J.R., and Harris C.C. 1994. Hepatitis B virus X protein inhibits p53 sequence-specific DNA binding, transcriptional activity, and association with transcription factor ERCC3. Proc. Natl. Acad. Sci. 91: 2230.

Wong C.M., Fan S.T., and Ng I.O. 2001. $\beta$-Catenin mutation and overexpression in hepatocellular carcinoma: Clinicopathologic and prognostic significance. Cancer 92: 136.

Wong N., Lai P., Lee S.W., Fan S., Pang E., Liew C.T., Sheng Z., Lau J.W., and Johnson P.J. 1999. Assessment of genetic changes in hepatocellular carcinoma by comparative genomic hybridization analysis: Relationship to disease stage, tumor size, and cirrhosis. Am. J. Pathol. 154: 37. 


\section{$8_{8}^{\infty} \mathrm{CSH} \&$ Cold Spring Harbor Symposia SYMPOSJA on Quantitative Biology}

\section{Generation and Analysis of Genetically Defined Liver Carcinomas Derived from Bipotential Liver Progenitors}

L. ZENDER, W. XUE, C. CORDÓN-CARDO, et al.

Cold Spring Harb Symp Quant Biol 2005 70: 251-261

Access the most recent version at doi:10.1101/sqb.2005.70.059

References This article cites 56 articles, 16 of which can be accessed free at: http://symposium.cshlp.org/content/70/251.full.html\#ref-list-1

License

Email Alerting Receive free email alerts when new articles cite this article - sign up in Service the box at the top right corner of the article or click here.

To subscribe to Cold Spring Harbor Symposia on Quantitative Biology go to: http://symposium.cshlp.org/subscriptions 